www.jmscr.igmpublication.org

Impact Factor 5.84

Index Copernicus Value: 71.58

ISSN (e)-2347-176x ISSN (p) 2455-0450

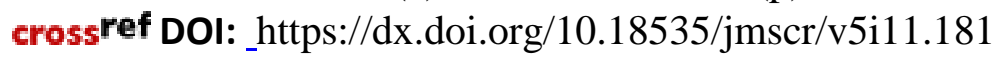

Journal Of Medical Science And Clinical Research

\title{
Cartilage Perichondrium Ring Graft versus Cartilage Perichondium Crescent Shaped Graft in Type One Tympanoplasty
}

\author{
Authors \\ Haitham El-farargy ${ }^{1}$, Mohamed Nasser ${ }^{2}$, Ahmed Gamea $^{3}$, Osama Albirmawy ${ }^{4}$ \\ ${ }^{1}$ ENT Department, Alhayat National Hospital, Jizan, Saudi Arabia \\ ${ }^{2,3,4}$ ENT Department, Tanta University, Tanta, Egypt
}

\begin{abstract}
To improve the functional and audiological results of Tympanoplasty, Ring graft was created but with some difficulty during insertion the graft medial to the handle of malleous. As a trial to overcome this disadvantage, we created a novel graft: cartilage-perichondrium-composite crescent shaped graft, which is put later all to the handle of malleous below the annulus (underlay). It has the same functional and audiological results of the ring graft but with a shorter time, making it superior in tympanoplasty type one

Keywords: tympanoplasty, cartilage, ring, crescent.
\end{abstract}

\section{Introduction}

The principal aims of tympanoplasty are to create an intact tympanic membrane and to restore functional hearing. ${ }^{16}$ The choice of operative technique and material for myringoplasty and tympanoplasty remains controversial. ${ }^{10}$ A variety of techniques have been described and are currently used, including overlay, underlay, sandwich, pegging, rosette and plugging. ${ }^{3,9,18}$ In addition, a wide variety of grafting materials have been used, in various autografts, homografts and allografts ${ }^{1-5}$ At the present time, autografts of temporalis fascia, veins, areolar tissue, periosteum and perichondrium, and involving either the overlay or underlay technique, appear to be most widely used. ${ }^{2,15,17}$ Each graft has its own, well known advantages and disadvantages. Some authors have suggested that soft tissue is an uncertain material with which to repair total perforations, especially those close to the annular region, and that more rigid transplant materials are needed. ${ }^{14}$ The aim of the work is to compare between the anatomical and audiological outcomes of primary type one tympanoplasty performed with a modified cartilageperichondrium composite ring graft (peripheral, ring shaped piece of cartilage attached to a sheet of perichondrium) to those performed with a modified cartilage-perichondrium composite crescent shaped graft (graft consisting of a peripheral, crescent shaped piece of cartilage attached to a sheet of perichondrium) as a novel technique.

\section{Patients and Methods}

This prospective study was carried out in ORLHNS Department in Tanta University Hospital .Study population was eighty consecutive cases undergoing type one tympanoplasty. Case enrollment started from October 2014 to Mars 
2015.The patients divided into 2 surgical groups,40 ears were grafted with ring shaped modified cartilage-perichondrium composite graft (group A). While the other 40 ears were grafted with modified cartilage-perichondrium crescent shaped graft as a novel technique (group B). The choice between was randomized and all patients were operated by the same surgeon All patients were approved to participate in the study by siging an informed consent. We included patients with dry tympanic membrane perforations of any size, with conductive hearing loss (air-bone gap not exceeding a mean of $25 \mathrm{~dB}$ ). We excluded cases with conductive hearing more than $25 \mathrm{db}$ or mixed hearing loss, Patients with concomitant mastoidectomy, ossiculoplasty or atelectasis. Any history of previous ear surgery and patients who did not continue the proper follow up. These patients were subjected to: Detailed history taking, complete general examination, complete ENT examination, otoscopic and microscopic examination of ears, audiological evaluation: pure tone audiometry with measurement of $\mathrm{ABG}$ at frequency 500,1000,2000, routine investigations for anesthesia. Any source of recurrent upper respiratory tract infections (e.g. sinusitis, allergic rhinitis or adenotonsillitis) was managed medically and/or surgically at least one month before surgery. Under general anesthesia with post-auricular approach was used in all cases, the tragal cartilage was taken, the perichondrium was left attached to the concave anterior side (lateral) of the cartilage, leaving the posterior perichondrium and the free edge of the tragal cartilage intact, to avoid tragal deformity. The graft was approximately $12-15 \mathrm{~mm}$ in diameter.

A circular piece of cartilage was cut from the centre of the graft using a number 15 scalpel blade or the sharp edge of an ear speculum. The cut, circular piece of cartilage was removed with gentle dissection. Care was taken to avoid laceration of the attached perichondrial sheet. In this way, it was possible to obtain a perichondrial sheet with attached cartilage ring frame. The cartilage ring was trimmed according to the periphery needed, taking care to preserve a good rim of firm, elastic, intact cartilage $(2-3 \mathrm{~mm})$, so that the size of the graft would be a little larger than the size of the membrane tensa. In crescent graft about 2-3 $\mathrm{mm}$ of the peripheral cartilaginous ring was removed to accommodate handle of malleus. After preparation of the middle ear and tympanic membrane, tympanoplasty proceeded as follows. both grafts were used as an underlay with their cartilage facing medially and perichondrium facing laterally. In the ring group, we put the graft medial to the handle of malleus. However, in the crescent group, we put the graft lateral to the handle of malleus, with the upper part of the graft accommodating it. In both groups, the graft is covered by the tympanic membrane remnant and the posterior tympano-meatal flap. Care was taken to place the graft well under the annulus, or in the bony annulus if the fibrous annulus was absent or removed. In both groups, the transparent perichondrium is in a central position. In both groups, Gelfoam was used to pack the external ear canal lateral to the reconstructed tympanic membrane without putting Gelfoam in the middle ear in both groups. The patients were discharged the next morning on a 10-day course of oral amoxicillin-clavulanic acid. At the end of the $10^{\text {th }}$ post-operative day, the Gelfoam was sucked from the external ear canal to avoid a granulomatous reaction. Three weeks later, the patients were encouraged to start doing gentle Valsalva manoeuvres. After the end of the eight ${ }^{\text {th }}$ week, if hearing results were available and the tympanic membrane looked clear, the patient was followed after three months. Successful graft acceptance was defined as full, intact healing of the tympanic membrane without perforation, retraction, lateralization or anterior blunting. Assessment of hearing improvement was based on the most recent audiogram available. Results were analyzed by comparing pre- and post-operative PTA ABGs at 0.5, 1, 2 and $3 \mathrm{kHz}$. The ABG change was defined as the difference between the pre- and post-operative ABGs. An ABG closure to within $20 \mathrm{~dB}$ was considered successful. 


\section{Results}

The total number of patients is 80 . The ring graft comprised 40 patients and the crescent graft comprised 40 patients .In ring graft group the age ranged from 13 years to 30 years with a mean age of $20.1 \pm 4.584$ years. In the crescent graft group the age ranged from 13 years to 35 years with mean age of $20.2 \pm 4.942$ years. There were 21 males ( 52 per cent) and 19 females( 48 per cent) in ring graft group. in crescent graft group, there were 19 males (48 per cent) and 21 females (52 per cent). The follow up period in ring graft group ranges from 3 to 6 months with a mean of $4.95 \pm$ 0.783 months. In crescent graft group ranges from 4 to 6 months with a mean of $5.025 \pm 0.768$ months. The graft success rate in ring graft was 87.5 per cent. Only 3 cases (7.5 per cent) developed postoperative perforations and 2 cases (5 per cent) developed postoperative lateralizetion. In crescent graft group the success rate was 92.5 per cent. Only 2 cases ( 5 per cent) developed postoperative perforation and only one case (2.5 per cent) developed lateralization. The difference in graft acceptance rates between the 2 groups is statistically insignificant ( $p>0.05$ ). In both groups there are successful hearing results. In ring graft group the mean of $\mathrm{ABG}$ preoperative is $(18.375 \pm$ 3.469) $\mathrm{dB}$ and the mean of ABG postoperative is $(7.625 \pm 5.429) \mathrm{dB}$ so the mean of improvement in $\mathrm{ABG}$ is $(10.75 \pm 5.495) \mathrm{dB}$. This difference is highly statistically significant $(\mathrm{p}<0.001)$. In crescent group the mean of $\mathrm{ABG}$ preoperative is(19.2 \pm 4.040$) \mathrm{dB}$ and the mean of $\mathrm{ABG}$ postoperative is $(8.5 \pm 5.454) \mathrm{dB}$ so the mean of improvement of ABG is $(10.7 \pm 4.392) \mathrm{dB}$ with a highly statistically significant difference $(\mathrm{p}<$ 0.001). Comparison of the two groups' audiological results not reveals any statistically significant difference $(\mathrm{p}>0.05)$. The mean time of operation in ring group was $(42.9 \pm 2.384)$ minutes. But in crescent group the mean time of operation is $(36.15 \pm 2.293)$ minutes. So there is significant difference in the time of operation between two groups as $(p<0.001)$, being shorter in group B.

\section{Discussion}

The goal of tympanoplasty is to reconstruct the tympanic membrane and the sound-conducting mechanism in a long-lasting way. Since the introduction of this procedure in the 1950s by Zoellner $^{11}$ and Wullstein ${ }^{19}$, numerous graft materials and placement techniques have been described to reconstruct the tympanic membrane. ${ }^{16}$ Temporalis muscle fascia and perichondrium remain the most frequently used materials, with a primary tympanoplasty closure rate of approximately 90 per cent. ${ }^{4,8}$ However, in pediatric populations (which have a high frequency of upper respiratory tract infection, Eustachian tube dysfunction and immune system immaturity, together with a greater incidence of advanced middle-ear pathology, retraction pockets, atelectasis and revision tympanoplasty, fascial and perichondrial grafts have been observed to undergo atrophy and subsequent failure in the post-operative period, regardless of placement technique. ${ }^{9}$ These results have encouraged otologists to use harder and more compatible graft materials. ${ }^{12}$ Graft cartilage harvested from the concha or tragus is easy to obtain, thick, hard, resistant to resorption and retraction, and convenient for shaping according to the size of the perforation and the nature of the middle-ear pathology. ${ }^{6}$ However, the risk of significant conductive hearing loss following cartilage tympanoplasty has always hampered routine acceptance of cartilage as a graft material. ${ }^{5}$ Using a stiff, opaque graft, such as cartilage for tympanic membrane reconstruction in cases of cholesteatoma, recurrent disease may be difficult to detect if the entire ear drum is reconstructed with cartilage. It is not advisable to reconstruct the entire tympanic membrane with cartilage in cases of cholesteatoma. ${ }^{7}$ In an attempt to increase the advantages of the cartilage-perichondrium composite graft while avoiding its disadvantages, Mansour et al. modified what is now termed a "cartilage-perichondrium composite ring graft". Removing most of the central cartilaginous disc while keeping a narrow $(2-3 \mathrm{~mm})$ peripheral rim 
with its normal harvest thickness makes the central part of the same thickness of normal tympanic membrane, this enabled rapid healing and no delay in hearing, and makes the central part translucent allowing good examination of the middle ear especially in the follow up in cases of cholesteatoma. Leaving peripheral 2-3 $\mathrm{mm}$ cartilaginous ring makes support of the graft especially in the absence of fibrous annulus and prevent shrinkage of the graft or formation of retraction pockets without effect on the middle ear space. ${ }^{1,13}$ In our study we use crescent-shaped cartilage-perichondrium composite graft as a novel technique. Like the ring graft, we removed the central cartilaginous part leaving a peripheral 2-3 $\mathrm{mm}$ of cartilage attached to a sheet of perichondrium and remove the upper $2-3 \mathrm{~mm}$ of the cartilage rim making crescent-shaped graft allowing accommo-dation of handle of malleus. We compare between the anatomical and functional results of the ring graft and the crescent-shaped graft in tympanoplasty type 1 in 80 patients with dry, non-cholesteatomatous ears and an intact ossicular chain, to eliminate any other factor that may affect outcomes ensuring that, the anatomical and functional results can only affected by graft material only with follow up period about six months. the patients are divided into 2 groups in a random manner and operated by the same surgeon. The graft success rate in ring graft was 87.5 per cent. only 3 cases $(7.5$ per cent) developed postoperative perforations and 2 cases (5 per cent) developed postoperative lateralizetion. In crescent graft group the success rate was 92.5 per cent. Only 2 cases( 5 per cent) developed postoperative perforation and only one case (2.5 per cent) developed lateralization. The difference in graft acceptance rates between the 2 groups is statistically insignificant ( $p>0.05)$. in both groups, the postoperative perforations treated by conservative method. In both groups there are successful hearing results. in ring graft group the mean of ABG preoperative is $(18.375 \pm 3.469)$ and the mean of $\mathrm{ABG}$ postoperative is $(7.625 \pm$ 5.429) so the mean of improvement in $A B G$ is
$(10.75 \pm 5.495)$.in crescent group the mean of ABG preoperative is $(19.2 \pm 4.040)$ and the mean of $\mathrm{ABG}$ postoperative is $(8.5 \pm 5.454)$ so the mean of improvement of $\mathrm{ABG}$ is $(10.7 \pm 4.392)$. so there is no significant difference in hearing results ( $\mathrm{p}>0.05$ ).

The mean time of operation in ring group is (42.9 $\pm 2.384)$ minutes. but in crescent group the mean time of operation is $(36.15 \pm 2.293)$ minutes. So there is significant difference in the time of operation between two groups as $(\mathrm{p}<0.001)$. the time of operation in crescent group is shorter. This can be due to difficult insertion of the ring graft under handle of malleus especially when it is medially retracted, but in the crescent-shaped graft, it can accommodate the handle of mallus making the time of operation shorter. Accommodation of the handle of maleus, makes good support for the graft, eliminating the necessity of placing Gelfoam in the middle ear to support the graft position, decreasing time of the operation .The absence of amino acid chains from disintegrating middle-ear Gelfoam would be expected to reduce the risk of middle-ear infection in the early post-operative period, and to promote earlier aeration of the middle-ear space. This, in combination with the early removal of the external canal Gelfoam, maximized the restoration of normal middle-ear and auditory canal physiology, and probably enhanced the ultimate anatomical and functional results.

\section{Conclusion}

The crescent-shaped graft has the same anatomical and audiological results of that of the ring graft, but with a significant shorter time of the operation. So, it is better to use the crescentshaped cartilage-perichondrium composite graft in tympanoplasty operation type 1 in different sizes and positions of perforations especially in medially retracted handle of malleous.

\section{References}

1. Albirmawy OA. Comparison between cartilage-perichondrium composite 'ring 
2. graft and temporalis fascia in type one tympanoplasty in children .J LaryngolOtol 2010;124:967-74

3. Atef A, Talaat N, Moseleh M, Safwat S. Effect of the thickness of the cartilage disk on the hearing results after perichondriumcartilage island flap tympanoplasty. ORL 2007;69:207-11

4. Derlacki EL. Residual perforation after tympanoplasty: office technique for closure. OtolaryngolClinNorth Am1982; 15:861-7

5. Dornhover J. Hearing results with cartilage tympanoplasty.

Laryngoscope 1997;107:1094-9

6. Dornhoffer J. Cartilage tympanoplasty: indications, technique, and outcomes in a 1,000-patient series. Laryngoscope 2003;112:1944-56

7. Dornhoffer JL. Cartilage tympanoplasty. Otolaryngol Clin North Am 2006;39:116176

8. Duckert LG, MullerJ, Makielski KH, Helms J. Composite autograft "shield" reconstruction of remnant tympanic membranes .Am j Otol 1995;16:21-6

9. Gerber MJ, Mason JC, Lambert PR. Hearing results after primary cartilage tympanoplasty.

Laryngoscope 2000;110:1994-99

10. Gibb AG, Chang SK. Myringoplasty (a review of 365 operations).J LaryngolOtol 1982;96:915-30

11. Gross CW, Bassila M, Lazar RH, Long TE, Stagner S. Adipose plug myringoplasty: an alternative to formal myringoplasty techniques in children. Otolaryngol Head Neck Surg 1989; 101:617-20

12. Kazikdas KC, Onal K, Boyraz I, Karabulut E. Palisade cartilage tympanoplasty for management of subtotal perforations; a comparison with temporalis fascia technique. Eur Arch Otorhinolaryngol 2007;264:985-9
13. Kerr AG, Byrene JET, Smyth GDL. Cartilage homografts in the middle ear: a long term histologic study. J LaryngolOtol 1973;87:1193-9

14. Mansour MH, Askar MH, AlbirmawyOA. Repair of tympanic membrane perforation using a modified cartilage -perichondrium composite ring graft. J LaryngolOtol 2006;120:952-4

15. Milewski C,Giannakopoulos N, Muller J, Schon F.Tragus perichondrium island transplant in middle ear surgery. Methods and results after 5 years [in German].HNO 1996;44:235-41

16. Ozbek C, Ciftci O, Tuna E, Yazkan O, Ozdem C. A comparison of cartilage palisades and fascia in type I tympanoplasty in children: anatomic and functional results. OtolNeurotol 2008;29:679-83

17. Rizer FM. Overlay versus underlay tympanoplasty. Part I:historical review of the literature. Laryngoscope 1997; 107:125

18. Page C, Charlet L, Strunski V. Cartilage tympanoplasty: postoperative functional results. Eur Arch Otorhinolaryngol 2008;265:1196-8

19. Pfattz CR, Griesemer C. a new biomaterial for tympanoplasty: preliminary report. Am J Otol 1985; 6:266-8

20. Zollner F. The principles of plastic surgery of the sound conducting apparatus. J laryngolOtol 1952;69:657-68. 